\title{
Safe and effective prescription of metformin in elderly patients with type 2 diabetes (T2DM)
}

\section{Perspective}

We live in an aging world, $8.5 \%$ of population around the world are aged 65 and over according to National Institute of Health ${ }^{1}$ and this is expected to be around $17 \%$ by the year 2050. Expectedly, as Abdelhafiz \& Sinclair $^{2}$ explain, diabetes incidence is increasing all over the world too due to aging, longer living population and higher rates of obesity. Especially in the developed countries, it is becoming more evident that diabetes is no longer a disease of the middle aged people but more of a disease of older people as result of higher life expectancy. This brings with it special issues and adds specific complications of "geriatric syndromes" such as decline in the physical status, cognitive functional decline, higher risk of falls and polypharmacy on top of the well-known vascular and other complications of diabetes seen in younger age groups.

National Institute for Health and Care Excellence ${ }^{3}$ in the UK recommends metformin as initial drug treatment of choice in patients with T2DM. Similarly American Diabetes Association (ADA) and
Volume 6 Issue 3 - 2018

Sami Ozturk

GPwSI Diabetes-CCG Board Member, UK

Correspondence: Sami Ozturk, GPwSI Diabetes-CCG Board Member, Southend, UK, Email sami483@gmail.com

Received: April II, 2018 | Published: June 25, 2018

the European Association for the Study of Diabetes (EASD) (2015) (Appendix 1) published a position statement on the management of T2DM putting metformin as first line drug of choice. Florez4 describes metformin as the "undisputed queen" of all type 2 diabetes drugs which tops all the professional society algorithms as first-line drug to be given as soon as patient is diagnosed with T2DM.

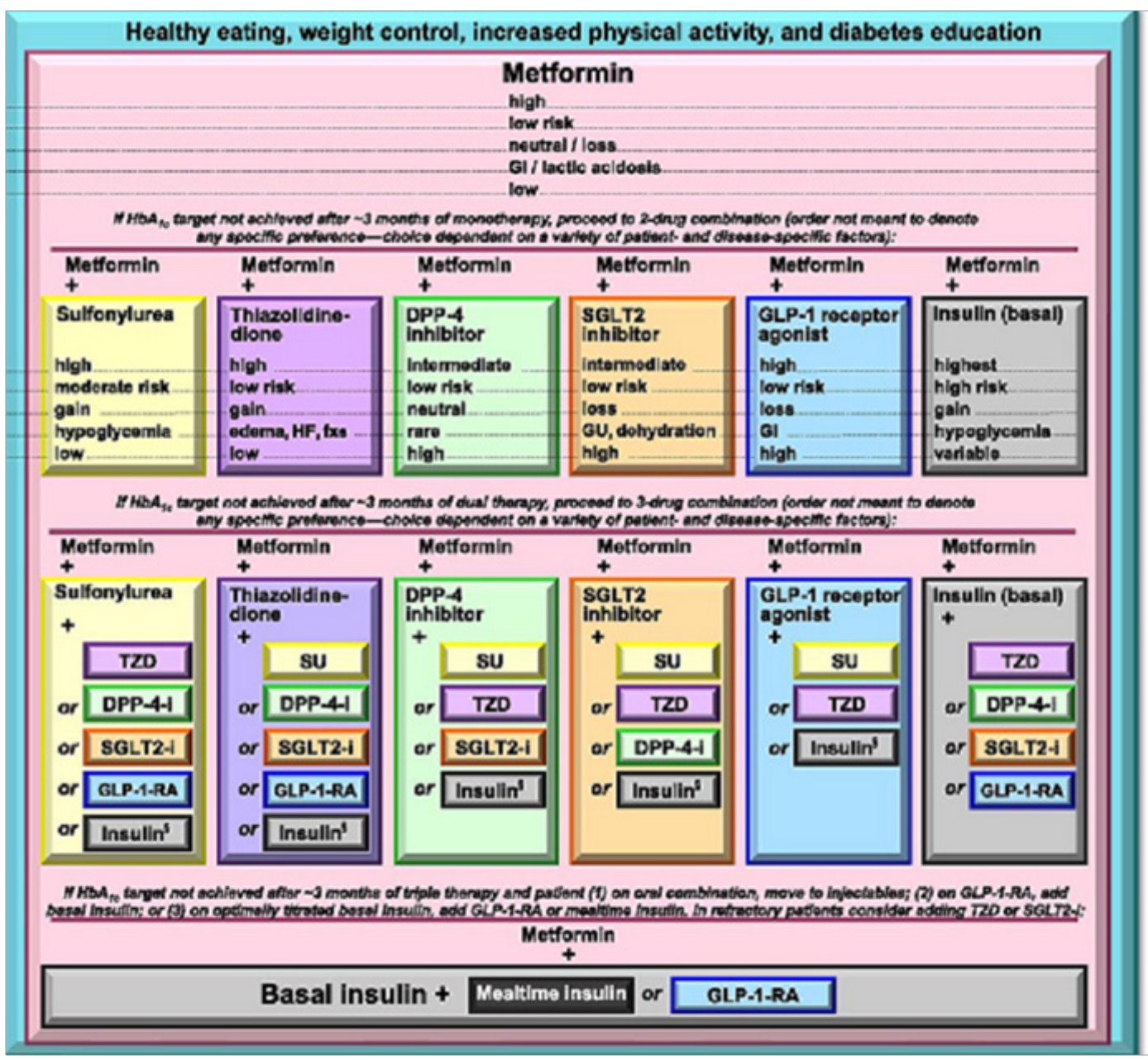

Appendix I American Diabetes Association (ADA) and the European Association for the Study of Diabetes (EASD) (20I5). 
Scheen \& Paquot ${ }^{5}$ however mention that elderly patients especially with diabetes prone to accumulate multiple comorbidities which are considered contraindications to the use of metformin, hence special consideration should be given when it is prescribed in this group.

Following considerations should be given when prescribing metformin in elderly patients with diabetes:

1. Elderly patients with diabetes are a distinct group and should have special consideration when it comes to prescribing. Individualised care with holistic approach is paramount

2. Metformin dosage should be reviewed according to renal functions, nutritional status of the patient and consider dose change or stop metformin use accordingly

3. Lactic acidosis is rare but could be fatal in elderly patients with diabetes; hence metformin use should be actively supervised in elderly patients where lactic acidosis risk is high.

4. Polypharmacy can make elderly diabetic patients more prone to lose weight, malnutrition hence it should be actively monitored.

5. Metformin use can lead to vitamin B12 deficiency which can have impact on elderly patient's cognitive and physical health

6. CHF is common in elderly patients, metformin use can continue but needs closer supervision

7. Gastrointestinal side effects of metformin are common. This could be primary at the initiation of metformin or secondary after metformin had been used for a while. Slow release metformin can be helpful if gastrointestinal side effects don't settle.
8. Sick day rules are important to educate the patient and the carers about to avoid potential harm

9. Although rare, hypoglycaemia can occur with metformin use in elderly and could be difficult to recognise. As result of recurrent hypoglycaemia, elderly patients' cognitive and physical health can deteriorate with subsequent frailty and raised mortality.

\section{Acknowledgments}

None.

\section{Conflict of interest}

The author declares there is no conflict of interest

\section{References}

1. National Institute of Health. World's older population grows dramatically. 2016.

2. Abdelhafiz AH, Rodriguez-Manas L, Morley JE, et al. (2015) Hypoglycemia in Older People-A Less Well Recognized Risk Factor for Frailty. Aging Dis. 6(2):156-167.

3. National Institute for Health and Clinical Excellence. Diabetes Type 2. 2015.

4. Florez, JC. The pharmacogenetics of metformin. Diabetologia. 2017;60(9):1648-1655.

5. Scheen AJ, Paquot N. Metformin revisited: A critical review of the benefit-risk balance in at-risk patients with type 2 diabetes. Diabetes and Metabolism. 2013;39(3):179-190. 